Original research article

\title{
Brain perfusion evaluated by perfusion-weighted magnetic resonance imaging before and after stenting internal carotid artery stenosis in asymptomatic and symptomatic patients
}

\author{
Maria Małowidzka-Serwińska ${ }^{a, *}$, Magdalena Żabicka $^{b}$, Adam Witkowski $^{c}$, \\ Zbigniew Chmielak ${ }^{c}$, Tomasz Deptuch ${ }^{c}$ \\ ${ }^{\text {a } U n i t ~ o f ~ N e u r o l o g y, ~ I n s t i t u t e ~ o f ~ C a r d i o l o g y, ~ W a r s a w, ~ P o l a n d ~}$ \\ ${ }^{\mathrm{b}}$ Department of Radiology, Military Institute of Medicine, Warsaw, Poland \\ ${ }^{\mathrm{c}}$ Department of Interventional Cardiology and Angiology, Institute of Cardiology, Warsaw, Poland
}

\section{A R T I C L E I N F O}

\section{Article history:}

Received 22 January 2015

Received in revised form

31 July 2015

Accepted 12 October 2015

Available online 24 October 2015

Keywords:

Magnetic resonance imaging

Perfusion-weighted imaging

Carotid artery stenosis

Carotid stenting

Cerebral hemodynamics

\begin{abstract}
A B S T R A C T
Purpose: To evaluate the brain perfusion with MRI perfusion weighted imaging (PWI) before and after ICA stenting in asymptomatic and symptomatic patients.

Materials and methods: PWI was performed 3-21 days before and 3 days after ICA stenting in 31 asymptomatic patients with ICA $>70 \%$ stenosis - Group I, and in 14 symptomatic patients with ICA $>50 \%$ stenosis - Group II. PWI was evaluated qualitatively and quantitatively in 5 cerebral territories with: mean transit time (MTT), cerebral blood volume (CBV) and cerebral blood flow (CBF). Mean values of perfusion parameters were measured before and after stenting $\triangle \mathrm{MTT}, \triangle \mathrm{CBV}, \triangle \mathrm{CBF}$ were calculated as subtraction of after-treatment values from those before treatment.

Results: In qualitative evaluation after ICA stenting perfusion was normalized in 21 patients $(80.8 \%)$ in Group I and in 8 patients $(80 \%)$ in Group II.

In quantitative estimation MTT decreased significantly after CAS on stented side vs. nonstented side in all examined patients regardless of the group, $p<0.05$. MTT decreased more in Group II than in Group I in all territories $(p<0.05)$ with the exception of temporal lobe. CBV and CBF have shown insignificant differences.

Conclusions:
\end{abstract}

1. In MRI the most useful parameters to assess brain perfusion are MTT and $\triangle \mathrm{MTT}$ : regardless whether patients are asymptomatic or symptomatic.

\footnotetext{
* Corresponding author at: Unit of Neurology Institute of Cardiology, Alpejska 42, 04-628 Warszawa, Poland. Tel.: +48 601851631; fax: +48223434506 .

E-mail address: m.malowidzka@ikard.pl (M. Małowidzka-Serwińska). http://dx.doi.org/10.1016/j.pjnns.2015.10.005 0028-3843/@ 2015 Polish Neurological Society. Published by Elsevier Sp. z o.o. All rights reserved.
} 
2. There were no significant differences in CBV and CBF after stenting in both groups of patients.

3. The positive effect of ICA stenting measured with decrease of MTT, CBV values and increase of $\mathrm{CBF}$ value is more prominent in symptomatic patients.

(C) 2015 Polish Neurological Society. Published by Elsevier Sp. z o.o. All rights reserved.

Keypoints:

Perfusion MRI can provide clinically useful information. The best indicator of perfusion changes is MTT. Decrease of MTT is more prominent in symptomatic patients.

\section{Introduction}

Internal carotid artery (ICA) stenosis can cause both acute ischemic stroke and chronic brain ischemia.

Severe ICA stenosis discriminates cerebral perfusion in the distal part of ICA vascularity - in vascularity territory of middle cerebral artery and/or anterior cerebral artery.

The increase of cerebral perfusion disturbances depends first of all on the rate of the stenosis increase, the efficiency of the circle of Willis, collateral circulation and also systemic blood pressure. It depends less, and in the opinion of some investigators does not depend at all, on carotid stenosis severity [1-3].

The longer carotid artery atherosclerosis lasts the more cerebral autoregulation is damaged, which in turn increases the stroke risk [4].

The findings of a growing number of randomized clinical studies show that ICA stenting improves cerebral perfusion. The changes of cerebral perfusion are seen just after intervention in symptomatic patients, while their influence is less pronounced in asymptomatic patients [3,5-8].

The reports on perfusion with MRI are not numerous. It especially refers to asymptomatic patients where the number of patients examined is insufficient to estimate credibly the value of stenting in primary stroke prophylaxis and risk for hyperperfusion syndrome, microhemorrhages or microstrokes producing the possibility of developing dementia in the future.

An open question is whether asymptomatic patients benefit approximately just as symptomatic patients do, and in which cases the risk of hyperperfusion syndrome exists after stenting.

In most patients brain perfusion after ICA dilatation increases by $20-40 \%$ over the baseline value. This increase takes place in the first few hours after intervention and is mostly asymptomatic $[9,10]$.

In some patients, most often 3-4 days after stenting, the increase of perfusion evaluated with computer tomography (CT) is above $100-200 \%$ of initial value found in the contralateral hemisphere, which results in cerebral hyperemia and clinical hyperperfusion syndrome [9].
In most reports, hyperperfusion syndrome occurs in 0-3\% of cases [3,9]. It is a rare but dangerous complication of carotid dilatation. This complication is considered as abnormal, worse cerebral vascular bed reactivity as a result of prolonged reduced perfusion on the stenosed artery side.

It is not more common in patients with severe ICA stenosis or coexisting contralateral ICA stenosis [9,11,12]. Clinical symptoms range from severe to almost none, through headache, nausea, vomiting, blurred vision, epileptic seizures, focal neurological symptoms to acute cerebral edema, intracranial hemorrhage. But also an opposite phenomenon is seen: in spite of clinical symptoms of hyperperfusion syndrome increased cerebral perfusion does not appear [9].

PWI and DWI with MRI help detect perfusion disturbances, ischemic changes at their earliest phase without changes in cerebral tissue, give an insight into stroke dynamics in vivo [13].

There are two commonly used methods to measure MRI perfusion [14-17]. The more popular is dynamic susceptibility contrast imaging (DCS-MRI). It uses rapid measurements of MRI signal change in the brain during the first pass of a paramagnetic contrast bolus injected intravenously. Either T1, T2 or T2* weighted sequences can be used. The deconvolution of an arterial input function is necessary to obtain the tissue response function and to produce maps of perfusion parameters: MTT, CBV and CBF. $\mathrm{T}_{0}$ (time to arrival) and TTP (time to peak) could be estimated to show the local variations of contrast arrival time. Usage of a standard dose $0.1 \mathrm{mmol} / \mathrm{kg}$ b.w. of high relaxivity contrast agent or a double dose of conventional contrast agent improve the signal to noise ratio $[15,18]$ resulting in high quality of obtained images.

The other technique is arterial spin labeling ASL. It is an endogenous tracer method in which the magnetically labeled protons in inflowing blood exchange with tissue protons producing the change of signal. The image of cerebral blood flow can be obtain by measuring signal changes between labeled and baseline unlabeled images.

The study of perfusion parameters before and after ICA stenting can bring the knowledge about pathophysiological factors of stroke, cerebral autoregulation disturbances in asymptomatic and symptomatic patients with ICA stenosis, help identify patients at risk for hyperperfusion syndrome $[3,11]$. The information from DWI, PWI imaging can be used in choosing treatment methods and evaluating complications [19-21].

DWI, PWI and their correlation with neurological state seem to allow physicians to detect a patient's profile, risk factors, characteristics predicting the onset of such complications as hyperperfusion syndrome, vegetative disturbances, and can improve patients' selection who will benefit from revascularization [22]. 


\section{The aim}

1. To assess qualitatively perfusion in asymptomatic and symptomatic patients with ICA stenosis before and after stenting in early phase.

2. To assess quantitatively perfusion before and in early phase after stenting with PWI (MTT, CBV, CBF) in homologous brain areas on the stented and non-stented sides in asymptomatic and symptomatic patients.

\section{Materials and methods}

The study was carried out with the approval of the Institutional Ethics Committee and a written informed consent was obtained from each patient.

\subsection{Subjects}

Group I included 31 patients (8 females, 23 males) with asymptomatic ICA stenosis; aged 52-79 years, mean 68.4 years. Perfusion qualitative and quantitative evaluations were performed in all participants.

Group II included 14 patients (5 females, 9 males) with symptomatic ICA stenosis; aged 50-77 years, mean 63.4 years. PWI was assessed qualitatively in 14 patients, quantitatively in 12 patients.

Inclusion criteria were:

- contraindications to ICA endarterectomy;

- lack of patient's consent for surgery;

- informed patient's consent for ICA stenting;

- lack of contraindications to MRI;

- Group I - asymptomatic, unilateral ICA stenosis 70-99\%; irrelevant stenosis $<50 \%$ of contralateral ICA;

- Group II - symptomatic ICA stenosis $>50 \%$ responsible for cerebral vascular incidents;

TIA, previous strokes - within 180 days before the study; stenosis of contralateral ICA even to $90 \%$.

Exclusion criteria were:

- ICA occlusion;

- absolute contraindication to dual antiplatelet therapy (gastrointestinal bleeding, acute hemorrhagic stroke, acetylsalicylic acid allergy);

- symptomatic $>50 \%$ ICA tenosis + severe heart insufficiency - NYHA IV class;

- left ventricular dysfunction - ejection fraction $<30 \%$;

- recent myocardial infarction - up to 30 days from attack;

- severe lung disease;

- kidney insufficiency - GFR $<50 \mathrm{ml} / \mathrm{min} \times 1.72 \mathrm{~m}^{2}$.

All interventions were performed with neuroprotection.

\subsection{Magnetic resonance imaging}

MRI scans were obtained 3-21 days before and 3 days after ICA stenting.
Imaging was performed with a $1.5 \mathrm{~T}$ scanner (Magnetom Avanto; Siemens) using of a standard quadrature head coil. The study included morphological evaluation, diffusionweighted imaging (DWI) and cerebral perfusion.

The scanning protocols included: axial T1-weighted images (spin-echo; $\mathrm{TR}=470 \mathrm{~ms}, \mathrm{TE}=11 \mathrm{~ms}$ ). T2-weighted images (spin-echo TR $=4750 \mathrm{~ms}, \mathrm{TE}=105 \mathrm{~ms}$ ), fluid-attenuated inversion recovery images (T2 TIRM TR: $9000 \mathrm{~ms}, \mathrm{TE}=89 \mathrm{~ms}$, $\mathrm{TI}=2500 \mathrm{~ms}$ ), diffusion-weighted images using single-shot echo-planar imaging sequence $(\mathrm{TR}=3200 \mathrm{~ms}$, TE $=101 \mathrm{~ms}, b$ values $=0,500,1000$ and $1500 \mathrm{~s} / \mathrm{mm}^{2}$ ), susceptibility weighted images (3D GR,TR $=49 \mathrm{~ms}, \mathrm{TE}=40 \mathrm{~ms}$ ).

PWI data were acquired using a T2*-weighted echo-planar dynamic volume imaging sequence (dynamic susceptibility contrast imaging) ( $\mathrm{TR}=1580 \mathrm{~ms}, \mathrm{TE}=41 \mathrm{~ms}, 5 \mathrm{~mm}$ slice/ $1.5 \mathrm{~mm}$ intersection gap, 50 series of 19 slices, total acquisition time $1.24 \mathrm{~min}$ ) after intravenous injection of standard dose $0.1 \mathrm{ml} / \mathrm{kg} / \mathrm{b} . \mathrm{w}$. of a high relaxivity paramagnetic contrast agent gadobenate dimeglumine (Gd-BOPTA, MultiHance Bracco) at $5 \mathrm{ml} / \mathrm{s}$ followed by a $20 \mathrm{ml}$ flush of saline with the use of an automatic injector [18].

Qualitative evaluation was made on the basis of perfusion maps created by software provided by the MR scanner manufacturer.

Quantitative evaluation was performed in Nordic ICE v 2.3.12 software with perfusion module of the Nordic Neurolab company. For this analysis the maps were created separately for each tested parameter: MTT, CBV, CBF.

MTT, mean transit time in seconds; CBV, cerebral blood volume in $\mathrm{ml} / 100 \mathrm{~g}$ tissue; $\mathrm{CBF}$, cerebral blood flow in $\mathrm{ml} /$ $100 \mathrm{~g}$ tissue/min.

\subsection{Image analysis}

Changes in 5 brain areas in the vascular territory of ICA [22] were compared for MTT, CBV, CBF; 1 and 4 - frontal lobe (basis and above fornix), 2 - temporal lobe, 3 - basal ganglia (head of caudate nucleus), 5 - parietal lobe.

Qualitative evaluation was made on the basis of colorcoded perfusion maps created by software provided by the MR scanner manufacturer. The rainbow scale of colors was used from red color for the highest value to violet for lowest - to compare the maps of the same parameter created for every patient. The assessment was based on the changes of colors and their intensity in the comparative regions of the brain.

\subsection{Quantitative evaluation}

The round shape regions of interest (ROI) area were: region no 1, 2, 4 and $5-452.03 \mathrm{~mm}^{2}$, region no $3-219.56 \mathrm{~mm}^{2}$. The location of particular ROI was drowned manually avoiding infarct lesions and sylvian fissure in similar location of the brain hemisphere in every patient and automatically copied parallel to the midline of the brain on the both hemispheres (Fig. 1). Measurements were made before and after stenting separately for each hemisphere. The mean values of perfusion parameters and their changes after treatment $(\triangle \mathrm{MTT}, \triangle \mathrm{CBV}$ and $\triangle \mathrm{CBF}$ ) calculated as the subtraction of after-treatment from before-treatment values were specified for all analyzed areas before and after ICA stenting. The results of perfusion 


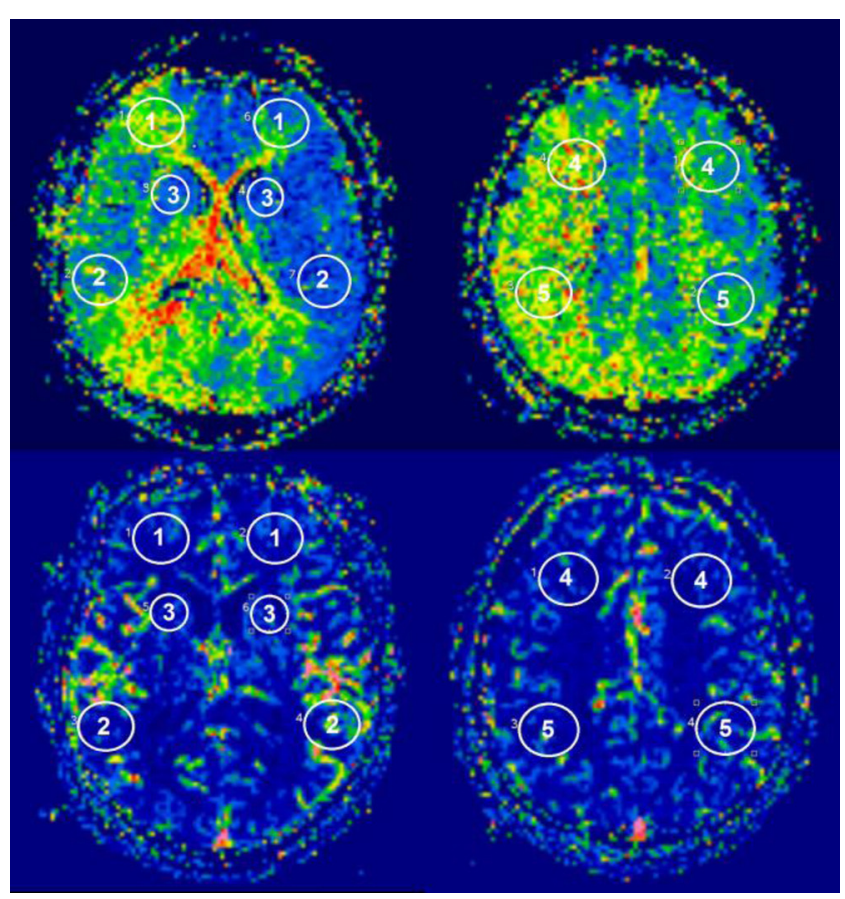

Fig. 1 - MRI perfusion maps of MTT (upper) and CBV (lower) with ROI placement.

parameters were analyzed in all patients and separately in Groups I and II. The values for the stented and non-stented side were compared in all patients and in Groups I and II separately.

Hyperperfusion syndrome criteria were: pulsating headache, nausea, vomiting, consciousness disturbance, epileptic seizure, focal neurological symptoms.

\subsection{Statistical analysis}

Analysis of variance ANOVA in repeated measurements were used to test for differences in categorical variables.

The 2-tailed probability $p$ value $<0.05$ was considered statistically significant.

\section{Results}

\subsection{Qualitative perfusion evaluation in Group I}

Initial PWI study was normal in 5 patients (16.1\%), impaired perfusion with varying degrees of severity in narrowed artery territory was present in 26 patients (83.9\%).

Perfusion was normalized after stenting in $21(80.8 \%)$ out of 26 patients. The total of 25 PWI studies was normal (80.6\%). Hyperperfusion was in 2 patients (6.6\%): in one case initial PWI was normal, the other presented impaired perfusion in initial examination.

In $4(13 \%)$ both PWI studies revealed the same perfusion deficit.

In $4(13 \%)$ cases the symptoms of clinical hyperperfusion syndrome were observed, mostly they were severe headaches: in 2 cases - impaired perfusion in initial PWI, normalization in control PWI, 1 case - both PWI studies were normal, 1 case impaired initial PWI, hyperperfusion in control PWI.

In 4 cases (13\%), during or several hours after intervention, TIA appeared with symptoms from the territory of expanded ICA. In 3 cases perfusion normalization was seen in control study, in 1 case both PWI studies were normal.

\subsection{Qualitative perfusion evaluation in Group II}

In $4(28.6 \%)$ patients, initial and after intervention PWI were normal. Out of 10 cases, impaired initial perfusion improved after stenting in 8 patients $(80 \%)$.

After ICA treatment in 2 cases of impaired initial perfusion there was no improvement in 1 patient, in the other the perfusion assessment was not possible because of hematoma.

In no case did perfusion in PWI increase clearly, while in 2 patients (14.3\%) clinical hyperperfusion syndrome occurred; in one patient severe neurological symptoms - aphasia and right hemiparesis; in the other, hematoma in the area of enlarged artery vascularity in the second day after stenting.

There was one TIA case in this group.

\subsection{Quantitative perfusion analysis in both groups of patients}

Analysis of perfusion parameters was carried out before and after ICA stenting in all of the patients independently of the side: stented and non-stented, The analysis of variance was made for the whole group of patients and independently in Group I and Group II and compared between groups.

Before stenting the initial values of MTT were higher in Group II in all assessed areas in comparison with Group I. Also the values of MTT in the stented hemisphere were higher than in contralateral hemisphere.

After intervention statistically significant decrease of MTT value (negative $\Delta \mathrm{MTT}$ ) was observed in symptomatic group in regions 1, 3, 4 and 5 comparing to asymptomatic group.

In region 2 - temporal lobe the change of the value was insignificant.

Statistically significant decrease of MTT value (negative $\Delta \mathrm{MTT}$ ) was also observed on stented side vs. non-stented side in all areas regardless of patient group.

The results of $\Delta \mathrm{MTT}$ are shown in Table 1 and Figs. 2 and 3.

The results of $\triangle \mathrm{MTT}$ in multivariable analysis are presented in Fig. 4.

In multivariable analysis between groups of patients despite visible differences of $\Delta \mathrm{MTT}$ values in Group II (symptomatic patients) in the stented hemisphere vs. nonstented one and in comparison with Group I (asymptomatic patients) the analyzed values were not statistically significant in all assessed areas.

The multivariable analysis of results of $\triangle \mathrm{CBV}$ and $\triangle \mathrm{CBF}$ are shown in Figs. 5 and 6.

No statistically significant changes of $\triangle \mathrm{CBV}$ were seen. The values of CBV decreased or remained unchanged, with the exception of an apparent decline in the $\triangle \mathrm{CBV}$ values in Group II in the affected hemisphere especially in the temporal lobe.

The values of $\triangle \mathrm{CBF}$ were insignificant; $\triangle \mathrm{CBF}$ increased or remained unchanged, but $\triangle \mathrm{CBF}$ values increased in Group II on the stented site, most visibly in the temporal lobe. 
Table 1 - $\Delta$ MTT in asymptomatic and symptomatic groups in stented and non-stented hemispheres in evaluated regions.

\begin{tabular}{|c|c|c|c|c|c|c|}
\hline$\Delta \mathrm{MTT}$ & Asymptomatic & Symptomatic & $p$ & Non-stented side & Stented side & $p$ \\
\hline$\triangle \mathrm{MTT} 1$ & -0.52 & -1.35 & 0.0194 & -0.57 & -1.26 & 0.0370 \\
\hline$\Delta$ MTT 2 & -0.45 & -1.05 & 0.0594 & -0.13 & -1.36 & 0.0002 \\
\hline$\triangle \mathrm{MTT} 3$ & -0.29 & -0.99 & 0.0081 & -0.29 & -1.01 & 0.0065 \\
\hline$\Delta \mathrm{MTT} 4$ & -0.26 & -1.24 & 0.0011 & -0.38 & -1.13 & 0.0116 \\
\hline$\triangle \mathrm{MTT} 5$ & -0.21 & -1.33 & 0.0011 & -0.18 & -1.35 & 0.0006 \\
\hline
\end{tabular}

$\Delta \mathrm{MTT}$ - in seconds, 1 - frontal lobe basis, 2 - temporal lobe, 3 - basal ganglia, 4 - frontal lobe above fornix, 5 - parietal lobe, $p$ - $p$ value.

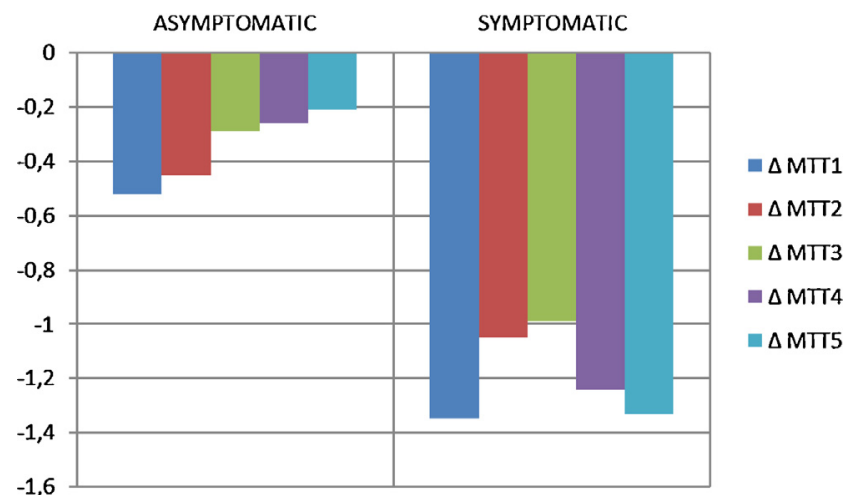

Fig. 2 - $\Delta$ MTT in seconds in evaluated brain areas in both groups of patients.

The analysis of PWI results in patients with hyperperfusion syndrome and TIA during ICA treatment.

Hyperperfusion syndrome occurred in 6 patients; 4 in Group I and 2 in Group II. In these patients no statistically significant differences were seen in all PWI parameters before and after stenting.

After ICA treatment, the decrease of mean MTT value (negative $\triangle \mathrm{MTT}$ ) by 0.1-0.9 s was observed in all patients on the stented side.

CBV mean value increased (positive $\triangle \mathrm{CBV}$ ) slightly in frontal lobe by $0.31 \mathrm{ml} / 100 \mathrm{~g}$, in the remaining areas decreased (negative $\Delta \mathrm{CBV}$ ) by $0.65-2.8 \mathrm{ml} / 100 \mathrm{~g}$.

CBF mean value increased (positive $\triangle \mathrm{CBF}$ ) only in basal ganglia on the stented side by $9.15 \mathrm{ml} / 100 \mathrm{~g} / \mathrm{min}$, in the remaining areas it decreased (negative $\Delta \mathrm{CBF}$ ) by $2.4-7.7 \mathrm{ml}$ / $100 \mathrm{~g} / \mathrm{min}$.

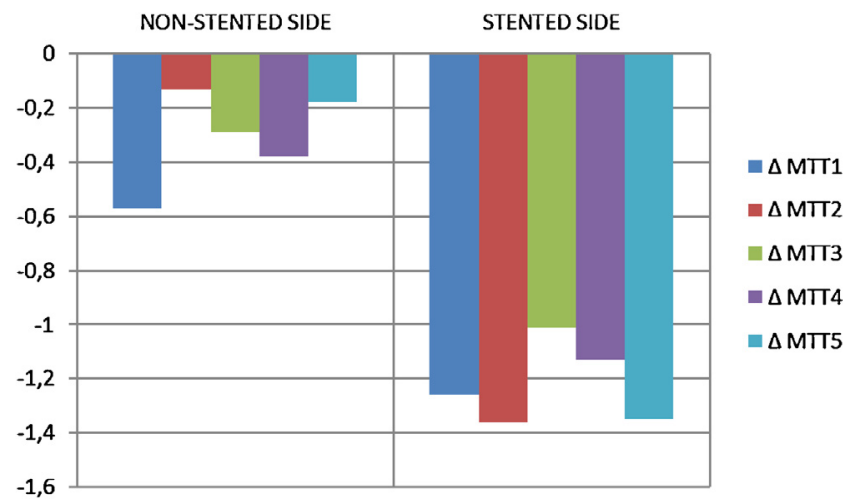

Fig. $3-\Delta M T T$ in seconds in evaluated brain areas in stented and non-stented hemisphere.
In 4 patients in Group I and one patient in Group II, TIA occurred during stenting. In none of these patients there were any statistically significant differences in PWI parameters. On the stented side MTT decreased (negative $\triangle \mathrm{MTT}$ ) by $0.5-1.31 \mathrm{~s}$, CBV decreased (negative $\Delta \mathrm{CBV}$ ) by $0.7-3 \mathrm{ml} / 100 \mathrm{~g}, \mathrm{CBF}$ increased (positive $\triangle \mathrm{CBF}$ ) in frontal lobe basis by $5.7 \mathrm{ml} / 100 \mathrm{~g} /$ min and in parietal lobe by $2.9 \mathrm{ml} / 100 \mathrm{~g} / \mathrm{min}$ and decreased in the other regions by $3.1-16.9 \mathrm{ml} / 100 \mathrm{~g} / \mathrm{min}$.

In Group I in DWI new small, ischemic lesions were found in $11(35.5 \%)$ cases, in Group II only in 2 (14.3\%) patients.

\section{Discussion}

Despite a long history of treating stenosed ICA, the literature assessing the effectiveness of this treatment method on the basis of perfusion evaluation is quite poor.

The available literature evaluates quantitatively brain perfusion before and after ICA stenting mostly with perfusion study by computed tomography (PCT) and in symptomatic patients.

There are few reports evaluating the usefulness of MRI in a brain perfusion study, and they are qualitative assessments [23].

Due to the relative variable dependent on the stability of the magnetic field of the apparatus, influence of protocol of examination and the type of contrast agent, the accuracy of measurement of perfusion parameters should be treated as relative.

Because of that fact only relative comparison of PWI results from different scanners can be possible (the absolute standard does not exist) and the relative comparison with the standards drawn up on the basis of studies PCT.

We chose the method of quantitative PWI assessment, which takes these limitations into account.

The changes of measured values were calculated as the results of the subtraction of after-treatment value from those before treatment and presented as $\triangle \mathrm{MTT}, \triangle \mathrm{CBV}$ and $\triangle \mathrm{CBF}$ and the multivariable model was used in statistical analysis.

Before stenting in symptomatic patients, MTT values were higher, while CBF values visibly lower in the affected hemisphere in relation to the contralateral side. It suggests that the decrease in cerebrovascular reactivity was the result of the fact that the cerebral vascular bed was no longer able to dilate [24].

After treatment MTT shortened statistically significant (negative $\Delta \mathrm{MTT}$ ) on the stented side in relation to the contralateral side independently of the studied group in all assessed areas. 


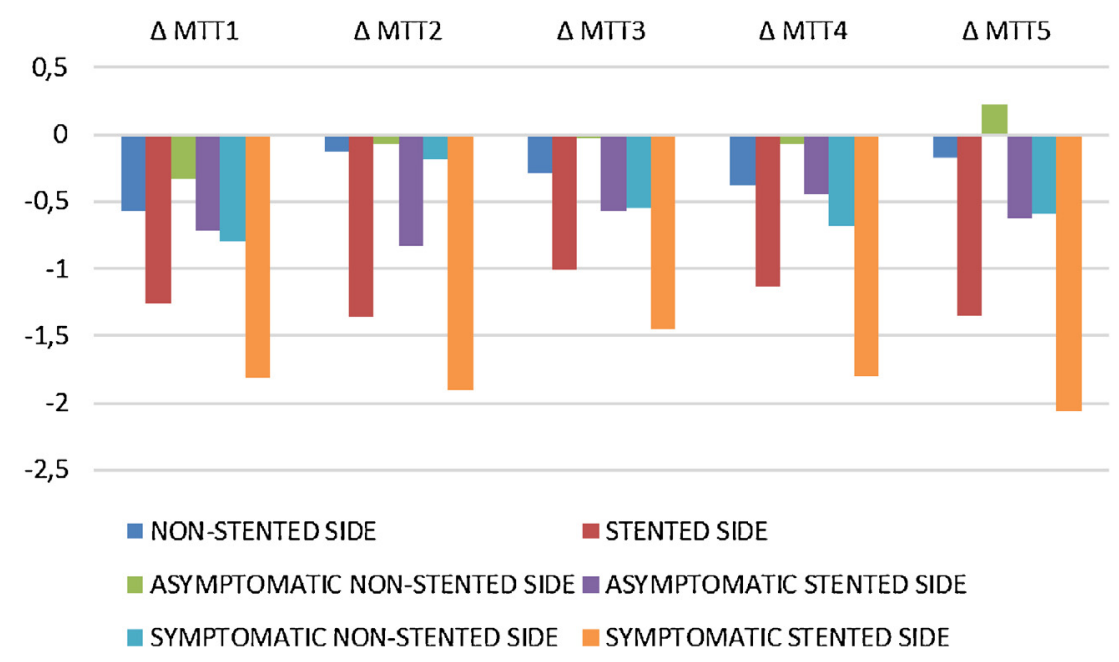

Fig. 4 - Multivariable analysis $\Delta$ MTT value in seconds in evaluated brain areas depending on non-stented and stented site and group of patients. The decreased of MTT are shown as negative $\triangle \mathrm{MTT}$.

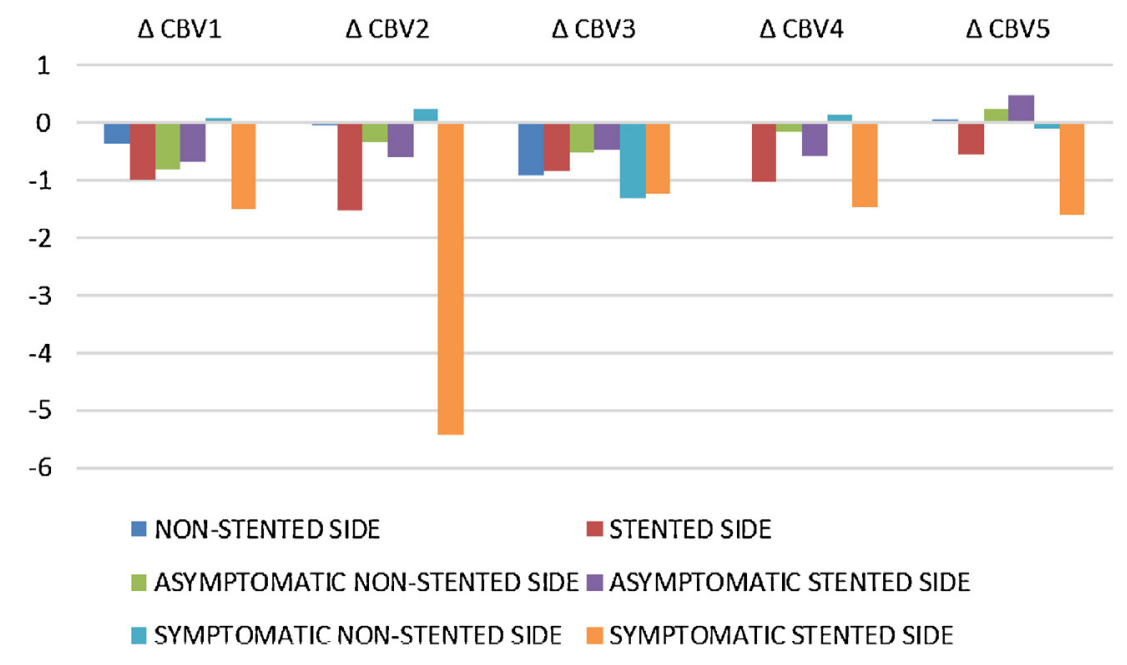

Fig. 5 - Multivariable analysis of $\triangle \mathrm{CBV}$ value in $\mathrm{ml} / 100 \mathrm{~g}$ in evaluated brain areas depending on non-stented and stented side and group of patients. The decrease of $\mathrm{CBV}$ is shown as negative $\triangle \mathrm{CBV}$.

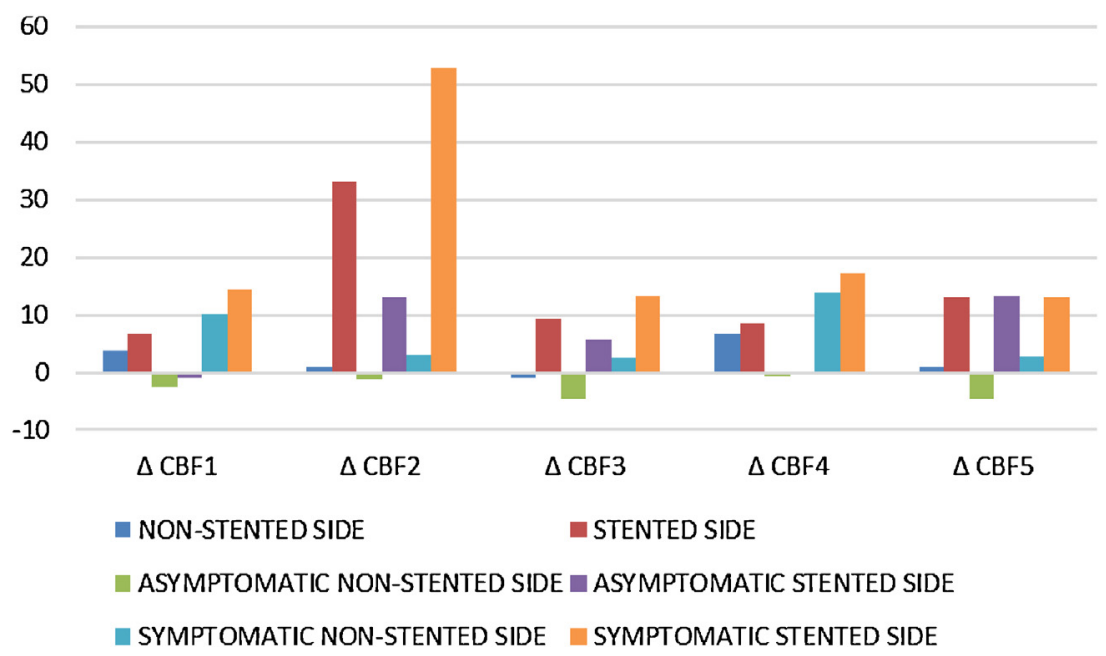

Fig. 6 - Multivariable analysis of $\triangle C B F$ value in $\mathrm{ml} / 100 \mathrm{~g} / \mathrm{min}$ in evaluated brain regions depending on non-stented and stented side and group of patients. The increase of $\mathrm{CBF}$ is shown as positive $\triangle \mathrm{CBF}$. 
This shortening of MTT (negative $\Delta \mathrm{MTT}$ ) was significantly higher in symptomatic group vs. asymptomatic group in all assessed areas except temporal lobe. This fact can be explained by measurement error or the possible impact of posterior cerebral artery supply of this region [22].

Comparing the values of $\triangle \mathrm{MTT}$ on stented and non-stented sides in symptomatic patients vs. asymptomatic patients no statistically significant changes of $\Delta \mathrm{MTT}$ were found but $\Delta \mathrm{MTT}$ values were visibly higher in symptomatic patient on both sides. The lack of statistical significance may be due to the small numbers of symptomatic patients.

In asymptomatic patients in parietal lobe on non-stented side $\triangle$ MTT slightly increase, but on stented side it slightly decrease. This after-treatment phenomenon is probably due to hemodynamic improvement on stented side so the higher MTT values on non-stented side were noted.

$\triangle \mathrm{CBV}$ and $\triangle \mathrm{CBF}$ values changed insignificantly in both groups of patients. $\triangle \mathrm{CBV}$ generally increased or remained unchanged on stented side and decreased or remained unchanged on non-stented side regardless of the group of patients.

But clear decrease of $\triangle \mathrm{CBV}$ in symptomatic group was observed on the stented side, especially in temporal lobe, which was accompanied by a marked increase in the value of $\triangle \mathrm{CBF}$. This shows a positive impact of stenting on cerebral hemodynamics in symptomatic patients. This phenomenon was not as much observed in asymptomatic group.

The results of our work are in many points, similar to the reports of other authors [25-28].

After treatment all reports observed reduced MTT, most researchers also pointed out the perfusion, measured by CBF indicator, increase.

Trojanowska et al. [25] in 50 subjects out of 74 with unilateral, symptomatic ICA stenosis (in 24 patients the other artery was occluded) found in their PCT study carried out in the third day after stenting an improvement in PWI parameters in $70 \%$ subjects, but the perfusion deficit symptoms remained in $30 \%$ patients. MTT shortened and CBF increased, CBV did not change after stenting. Before and after treatment both $\mathrm{CBF}$ and CBV were lower in the stented hemisphere vs. the non-stented one.

Gaudiello et al. [26] in 20 subjects, both symptomatic (15 cases) and asymptomatic (5 cases) found in PCT in the seventh day after treatment of stenosed ICA significant MTT shortening with simultaneous CBF increase on the stented side. Yet the values of these indicators did not change significantly in the non-stented side. Absolute values of these parameters were applied in the assessment.

Duan et al. [27] in 20 patients with symptomatic ICA stenosis found in PCT carried out in the seventh day after ICA treatment a significant $\mathrm{rCBF}$ increase and rMTT shortening in the vascular area of dilated ICA, but not in basal ganglia.

There were no significant changes in rCBV. Relative values of perfusion were calculated by comparing absolute values in symptomatic hemisphere to absolute values in asymptomatic hemisphere.

Szarmach et al. obtained slightly different results [28]. In assessing CT perfusion (absolute values) in 20 symptomatic subjects with ICA stenosis they found in the study made 4-8 weeks after stenting ICA, MTT shortening on the treated side without statistically significant changes in $\mathrm{CBV}$ and $\mathrm{CBF}$ values.

Mercel and all [29] comparing relative values (obtained by comparing treated to untreated hemisphere) of perfusion parameters in CT in 31 symptomatic and 14 asymptomatic patients found after treatment a statistically significant change in MTT values between the treated and untreated hemispheres (dMTT) only in symptomatic patients. In asymptomatic this parameter did not change significantly. Similar to our results the dMTT was also higher in symptomatic patients, but this difference was not significant. They thought this finding confirmed the presence of differences in cerebral hemodynamic between symptomatic and asymptomatic patients. The ratio of CBV (rCBV) between the abovementioned hemispheres decreased and the ratio of $\mathrm{CBF}(\mathrm{rCBF})$ increased.

In 14 asymptomatic patients they found only a significant rCBF increase after treatment. By comparing both groups before stenting they observed significantly lower rCBF values in symptomatic patients. The drop in rCBV value after intervention was significantly lower in symptomatic subjects vs. asymptomatic patients.

In our study this trend to more prominent decrease of CBV and increase of CBF (lower values before treatment) after treatment in symptomatic subjects was also observed showing the differences in hemodynamic status between symptomatic and asymptomatic patients.

Waaijer et al. [30] in patients with unilateral symptomatic carotid stenosis reported that MTT was the most useful and reproducible parameter of perfusion. Quantitative analysis of CBV and CBF values should be interpreted with caution.

Piñero et al. [31] examining perfusion by DSC-MRI in 33 patients with severe ICA stenosis found before and on the first day after treatment statistically significant increase of TTP (time to peak) and $T_{0}$ arrival time $\left(T_{0}\right)$ on the stenosed side vs. contralateral side. The values of this parameters normalized 30 days after treatment.

Tavares et al. [32] in 21 patients with severe ICA ( $\geq 60 \%)$ stenosis in MRI contrast perfusion made $12 \mathrm{~h}$ before and $72 \mathrm{~h}$ after ICA stenting found the hemodynamic improvement on the stented side described by statistically significant decrease of MTT $(p<0.001)$ and significant decrease of TTP $(p=0.019)$. This results were obtained for the relative values of perfusion calculated by comparing absolute values in symptomatic hemisphere to absolute values in asymptomatic one.

We did not analyze the TTP and $\mathrm{T}_{0}$ value in our study. TTP and $T_{0}$ concern to the vascular phase of perfusion, while we chose to analyze the tissue response parameters of perfusion as the main object of our study. The values of TTP and $T_{0}$ mainly depend on the blood supply and contrast dispersion and in to some degree on MTT value and are a good indicators of the severity of ICA stenosis. They do not describe independently the real conditions of hemodynamic status of brain tissue, which could be influenced by the contralateral blood supply via circle of Willis and auto regulatory processes [33]. 
The main role of cerebral autoregulation and the efficiency of contralateral blood supply were pointed out in works describing the lack of straight dependence of the severity of ICA stenosis on brain perfusion status [1-3].

Chang et al. [34] in DCS-MRI analyzed the change of CBF in patients with severe ( $270 \%)$ ICA stenosis before and on days 3-5 after stenting in comparing to cerebral vasoreactivity (CVR) representing the reserve capacity of cerebral perfusion. CVR was evaluated before treatment by fMRI using BOLD (blood oxygen level-dependent) technique. They found the statistically significant higher increase of CBF on stented side in short time after treatment in patients with impaired CVR. Impairment of CVR is reported as an independent risk factor for ischemic stroke and TIA in patients with critical carotid stenosis or occlusion and slightly higher risk for hyperperfusion syndrome after stenting. We observed a similar phenomenon of higher CBF increase in symptomatic patients after stenting, but not in the whole group with hyperperfusion symptoms.

This finding could be an indicator of more severe impairment of brain autoregulation in symptomatic patients. This study indicate how important is the proper qualification to the ICA stenting to prevent the patients from complications after procedure.

In our group with hyperperfusion syndrome, the perfusion values obtained met the criteria for increased brain perfusion [35]. We did not notice, (cited in the literature) the appearance of risk factor of hyperperfusion syndrome as increased MTT before treatment by at least $3 \mathrm{~s}$ in relation to the contralateral side [36].

In patients with TIA during treatment the most important is visible shortening of MTT accompanied by a drop of CBV value and a slight increase or decrease (depending of analyzed region) in $\mathrm{CBF}$ at the same time. Clinical symptoms, however, can result from the kind of neuroprotection used during the endovascular procedure itself or from microembolisms.

The results tend toward the following conclusions:

1. In MRI the most useful parameters to assess brain perfusion are MTT and $\triangle \mathrm{MTT}$ : regardless whether patients are asymptomatic or symptomatic.

2. There were no statistically significant differences in CBV and $\mathrm{CBF}$ after stenting in both groups of patients possibly due to the efficiency of cerebral autoregulation but this phenomenon was more pronounced in asymptomatic patients.

3. The positive effect of ICA stenting measured with decrease of MTT, CBV values and increase of CBF value is more prominent in symptomatic patients.

4. Slightly decreased MTT, decreased CBV and increased CBF in basal ganglia in patients with hyperperfusion syndrome can attest to the effectiveness of ICA stenting and TIA can be an indicator of procedure complication itself.

\section{Conflict of interest}

We wish to confirm that there are no known conflicts of interest associated with this study.

\section{Acknowledgement and financial support}

We wish to confirm that there has been no financial support for this work.

\section{Ethics}

The work described in this article has been carried out in accordance with The Code of Ethics of the World Medical Association (Declaration of Helsinki) for experiments involving humans; Uniform Requirements for manuscripts submitted to Biomedical journals.

\section{R E F E R E N C E S}

[1] Wojczal J. TCD and perfusion CT in patients before and after carotid artery stenting. Polski Przegląd Neurologiczny 2008;4(Suppl A):64.

[2] Reiche W, Schäfer R, Müller M, Hermes M. Cerebral perfusion reserve and collateral circulation in patients with internal carotid stenosis. Radiologe 1997;37(11):891-8.

[3] Ko NU, Achrol AS, Chopra M, Saha M, Gupta D, Smith WS, et al. Cerebral blood flow changes after endovascular treatment of cerebrovascular stenoses. AJNR Am J Neuroradiol 2005;26:538-42.

[4] Reinhard M, Roth M, Müller T, Czosnyka M, Timmer J, Hetzel A. Cerebral autoregulation in carotid artery occlusive disease assessed from spontaneous blood pressure fluctuations by the correlation coefficient index. Stroke 2003;34(9):2138-44

[5] Reinhard M, Roth M, Müller T, Guschlbauer B, Timmer J, Czosnyka M, Hetzel A. Effect of carotid endarterectomy or stenting on impairment of dynamic cerebral autoregulation. Stroke 2004;35(6):1381-7.

[6] Wilkinson ID, Griffiths PD, Hoggard N, Cleveland TJ, Gaines PA, Macdonald S, et al. Short-term changes in cerebral microhemodynamics after carotid stenting. AJNR Am J Neuroradiol 2003;24(8):1501-7.

[7] Tang SC, Huang YW, Shieh JS, Huang SJ, Yip PK, Jeng JS. Dynamic cerebral autoregulation in carotid stenosis before and after carotid stenting. J Vasc Surg 2008;48(1): 88-92.

[8] Sfyroeras G, Arsos G, Karkos CD, Liasidis C, Spyridis C, Boundas D, et al. Interhemispheric asymmetry in brain perfusion before and after carotid stenting: a $99 \mathrm{~m} \mathrm{Tc}$ HMPAO SPECT study. J Endovasc Ther 2006;13(6):729-37.

[9] Jędrzejewska J, Kobayashi A. Hyperperfusion syndrome. Terapia 2007;15(2):26-31.

[10] Van Laar PJ, Hendrikse J, Mali WP, Moll FL, van der Worp HB, van Osch MJ, et al. Altered flow territories after carotid stenting and carotid endarterectomy. J Vasc Surg 2007;45 (6):1151-61.

[11] Fukuda T, Ogasawara K, Kobayashi N, Komoribayashi N, Endo $\mathrm{H}$, Inoue T, et al. Prediction of cerebral hyperperfusion after carotid endarterectomy using cerebral blood volume measured by perfusion-weighted MR imaging compared with single-photon emission CT. AJNR Am J Neuroradiol 2007;28(4):737-42.

[12] Ascher E, Markevich N, Schutzer RW, Kallakuri S, Jacob T, Hingorani AP. Cerebral hyperperfusion syndrome after carotid endarterctomy; predictive factors and hemodynamic changes. J Vasc Surg 2003;37(4):769-77. 
[13] Rosenberger R, Wojtek P, Konopka M, Pieniążek P, Bogusz I, Sąsiadek M. Clinical applications of perfusion computed tomography, diffusion-weighted magnetic resonance and perfusion - weighted magnetic resonance imaging in detection of early lesions in ischemic stroke. Udar Mózgu 2004;6(2):71-8.

[14] Petrella JR, Provenzale JM. MR perfusion imaging of the brain: techniques and applications. AJR Am J Roentgenol 2000;175:207-19.

[15] Keston P, Murray AD, Jackson A. Cerebral perfusion imaging using contrast-enhanced MRI. Clin Radiol 2003;58(7):505-13.

[16] Polloc JM, Tan H, Kraft RA, Whitlow CT, Burdette JH, Maldjian JA. Arterial spin labeled MRI perfusion imaging: clinical applications. Magn Reson Imaging Clin N Am 2009;17(2):315-38.

[17] Essig M, Shiroishi MS, Nguyen TB, Saake M, Provenzale JM, Enterline D, et al. Perfusion MRI: the five most frequently asked technical questions. AJR Am J Roentgenol 2013;200 (1):24-34.

[18] Cotton F, Hermier M. The advantage of high relaxivity contras agents in brain perfusion. Eur Radiol 2006;16(Suppl 7):M16-26.

[19] Restrepo L, Wityk RJ, Grega MA, Borowicz Jr L, Barker PB, Jacobs MA, et al. Diffusion- and perfusion-weighted magnetic resonance imaging of the brain before and after coronary artery bypass grafting surgery. Stroke 2002;33:2909-15.

[20] Skjelland M, Krohg-Sørensen K, Tennøe B, Bakke SJ, Brucher R, Russell D. Cerebral microemboli and brain injury during carotid artery endarterectomy and stenting. Stroke 2009;40 (1):230-4.

[21] Bonati LH, Jongen LM, Haller S, Flach HZ, Dobson J, Nederkoorn PJ, et al. ICSS-MRI Study Group. New ischaemic brain lesions on MRI after stenting or endarterectomy for symptomatic carotid stenosis: a substudy of the International Carotid Stenting Study (ICSS). Lancet Neurol 2010;9(4):353-62.

[22] Gauvrit JY, Delmaire C, Henon H, Debette S, Al Koussa M, Leys D, et al. Diffusion/perfusion-weighted magnetic resonance imaging after carotid angioplasty and stenting. J Neurol 2004;251(9):1060-7.

[23] Van der Zwan A, Hillen B. Review of the variability of the territories of the major cerebral arteries. Stroke 1991;22:1078-84.

[24] Soinne L, Helenius J, Tatlisumak T, Saimanen E, Salonen O, Lindsberg PJ, Kaste M. Cerebral hemodynamics in asymptomatic and symptomatic patients with high-grade carotid stenosis undergoing carotid endarterectomy. Stroke 2003;34(7):1655-61.

[25] Trojanowska A, Drop A, Jargiełło T, Wojczal J, SzczerboTrojanowska M. Changes in cerebral hemodynamice after carotid stenting: evaluation with CT perfusion studies. J Neuroradiol 2006;33:169-74.

[26] Gaudiello F, Colangelo V, Bolacchi F, Melis M, Gandini R, Garaci FG, et al. Sixty-four-section CT cerebral perfusion evaluation in patients with carotid artery stenosis before and after stenting with a cerebral protection device. AJNR Am J Neuroradiol 2008;29:919-23.

[27] Duan Y, Li G, Yang Y, Li J, Huang H, Wang H, et al. Changes in cerebral hemodynamics after carotid stenting of symptomatic carotid artery. Eur J Radiol 2012;81(4):744-8.

[28] Szarmach A, Halena G, Buczny J, Studniarek M, Markiet K, Szurowska E, et al. Evaluation of changes in the parameters of brain tissue perfusion in multi-slice computed tomography in patients after carotid artery stenting. Pol J Radiol 2011;76(3):7-15.

[29] Merckel LG, Van der Heijden J, Jongen LM, van Es HW, Prokop M, Waaijer A. Effect of stenting on cerebral CT perfusion in symptomatic and asymptomatic patients with carotid artery stenosis. AJNR Am J Neuroradiol 2012;33 (2):280-5.

[30] Waaijer A, van der Schaaf IC, Velthuis BK, Quius M, van Osch MJ, Vonken EP, et al. Reproducibility of quantitative CT brain perfusion measurements in patients with symptomatic unilateral carotid artery stenosis. Am J Neuroradiol 2007;28(5):927-32.

[31] Piñero P, Gonzáles A, Moniche F, Martinez E, Cayuela A, Gonzáles-Marcos J-R, et al. Progressive changes in cerebral perfusion after carotid stenting: a dynamic susceptibility contrast perfusion weighted imaging study. J Neurointervent Surg 2014;6:527-32.

[32] Tavares A, Caldas JG, Castro CC, Puglia Jr P, Frudit ME, Barbosa LA. Changes in perfusion-weighted magnetic resonance imaging after carotid angioplasty with stent. Interv Neuroradiol 2010;16(2):161-9.

[33] Calamante F, Christensen S, Desmond PM, Østergaard L, Davis SM, Connelly A. The physiological significance of the time-to-maximum ( $T_{\max }$ ) parameter in perfusion MRI. Stroke 2010;41:1169-74.

[34] Chang TY, Liu HL, Lee TH, Kuan WC, Chang CH, Wu HC, et al. Change in cerebral perfusion after carotid angioplasty with stenting is related to cerebral vasoreactivity: a study using dynamic susceptibility-weighted contras enhanced MR imaging and functional MR imaging with breath-hold paradigm. AJNR Am J Neuroradiol 2009;30:1330-6.

[35] Moulakakis KG, Mylonas SN, Sfyroeras GS, Andrikopoulos V. Hyperperfusion syndrome after carotid revascularization. J Vasc Surg 2009;49(4):1060-8.

[36] Tseng YC, Hsu HL, Lee TH, Hsieh IC, Chen CJ. Prediction of cerebral hyperperfusion syndrome after carotid stenting: a cerebral perfusion computed tomography study. J Comput Assist Tomogr 2009;33(4):540-5. 